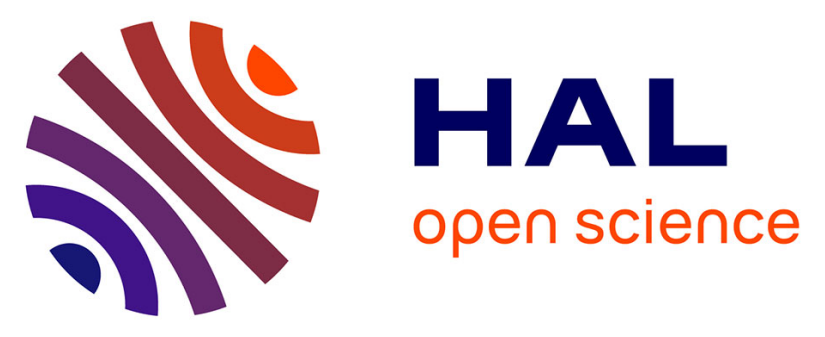

\title{
Ge-rich SiGe waveguides for supercontinuum generation in the mid-IR
}

Joan Manel Ramirez, Samuel Serna, Vladyslav Vakarin, Qiankun Liu, Jacopo

Frigerio, Andrea Ballabio, Xavier Le Roux, Laurent Vivien, Eric Cassan,

Giovanni Isella, et al.

\section{To cite this version:}

Joan Manel Ramirez, Samuel Serna, Vladyslav Vakarin, Qiankun Liu, Jacopo Frigerio, et al.. Gerich SiGe waveguides for supercontinuum generation in the mid-IR. Silicon Photonics: from Fundamental Research to Manufacturing, Apr 2018, Strasbourg, France. pp.24, 10.1117/12.2306873. hal-02362461

\section{HAL Id: hal-02362461 \\ https://hal.science/hal-02362461}

Submitted on 13 Nov 2019

HAL is a multi-disciplinary open access archive for the deposit and dissemination of scientific research documents, whether they are published or not. The documents may come from teaching and research institutions in France or abroad, or from public or private research centers.
L'archive ouverte pluridisciplinaire HAL, est destinée au dépôt et à la diffusion de documents scientifiques de niveau recherche, publiés ou non, émanant des établissements d'enseignement et de recherche français ou étrangers, des laboratoires publics ou privés. 


\title{
Ge-rich SiGe waveguides for supercontinuum generation in the mid-IR
}

\author{
J. M. Ramirez ${ }^{1, *}$, S. Serna ${ }^{1,2}$, V. Vakarin ${ }^{1}$, Q. Liu ${ }^{1}$, J. Frigerio ${ }^{3}$, A. Ballabio ${ }^{3}$, X. Le Roux ${ }^{1}$, L. Vivien ${ }^{1}$, \\ E. Cassan ${ }^{1}$, G. Isella ${ }^{3}$, N. Dubreuil ${ }^{2}$, D. Marris-Morini ${ }^{1}$ \\ ${ }^{1}$ Centre de Nanosciences et de Nanotechnologies, CNRS, Univ. Paris-Sud, Université Paris-Saclay, \\ C2N - Orsay, 91405 Orsay cedex, France
}

${ }^{2}$ Laboratoire Charles Fabry, Institut d'Optique Graduate School, CNRS, Université Paris Saclay, 2 Avenue Augustin Fresnel, 91127 Palaiseau cedex, France

${ }^{3}$ L-NESS, Dipartimento di Fisica, Politecnico di Milano, Polo di Como, Via Anzani 42, 22100 Como, Italy

*e-mail : joan-manel.ramirez@c2n.upsaclay.fr

\begin{abstract}
The third-order nonlinear parameter of Ge-rich $\mathrm{SiGe}$ waveguides are experimentally retrieved using a bi-directional top hat $\mathrm{D}$-scan at $\lambda=1.58 \mu \mathrm{m}$. The obtained values are then used to fit the theoretical equation, providing promising values in the mid-IR, where the nonlinear effects are no longer limited by two-photon absorption. New Ge-rich SiGe waveguide designs are provided to exploit the nonlinear properties in the mid-IR, showing a flat anomalous dispersion over one octave spanning from $\lambda=3$ $\mu \mathrm{m}$ to $\lambda=8 \mu \mathrm{m}$ and a $\gamma$ parameter that decreases from $\gamma=10 \mathrm{~W}^{-1} \mathrm{~m}^{-1}$.
\end{abstract}

\section{Introduction}

Mid-infrared (mid-IR) photonics is meant to lead to key advances in several disciplines including molecular sensing, early medical diagnosis or secure communications, among others. In particular, the combination of photonic structures where the light-matter interaction is enhanced with a wide transparent platform able to exploit the large number of absorption bands located along the mid-IR spectrum $(2-20 \mu \mathrm{m})$ is of high interest to develop label-free molecular sensors with unprecedented sensitivity. For that, several photonic platforms are currently being explored, including chalcogenide glasses, silicon-on-insulator, silicon-on-silicon nitride, germanium-on-silicon, germanium-on-silicon nitride, silicon-on-sapphire, SiGe alloys-on-silicon, aluminum nitride-on-insulator or suspended $\mathrm{Si}$ membranes, among others. In that regard, Si-based platforms are of particular interest due to their compatibility with standardized CMOS manufacturing tools, paving the way towards the monolithic integration of microelectronic drivers with photonic circuits operating at deep mid-IR wavelengths. For that purpose, the use of SiGe alloys to build such new mid-IR photonic platform shows a strong potential since it permits an accurate control of the optical properties by fine tuning the $\mathrm{Ge}$ concentration along the growth direction. Moreover, by combining high top Ge concentrations and a proper waveguide engineering the overlap of the propagating mode with the Si-rich area can be effectively minimized, hence remaining confined in the Ge-rich region of the waveguide cross-section. As a consequence, broadband low-loss performance at operating wavelengths far beyond the silicon multi-phonon absorption onset can be accomplished, providing access to a wider palette of substances to be potentially analyzed.

In line with such novel mid-IR integrated sensing platform, there is also a need for efficient broadband mid-IR sources able to cover the mid-IR wavelength range of interest in an efficient and compact manner. A plausible solution is to exploit the third order nonlinearity in Ge for supercontinuum generation, leveraging from the large nonlinear Kerr index $\left(\mathrm{n}_{2}\right)$ in Ge and the absence of two-photon absorption beyond $3.17 \mu \mathrm{m}$, which is represented by the $\beta_{\text {TPA }}$. Indeed, several demonstrations of 
supercontinuum generation have already been accomplished so far using SiGe alloys with moderate Ge concentration. Nevertheless, little exploration of these key parameters has been carried out using Ge-rich SiGe alloys. Moreover, there is no experimental data related to the nonlinear optical coefficient of these Ge-rich $\mathrm{Si}_{1-\mathrm{x}} \mathrm{Ge}_{\mathrm{x}}$ alloys.

Thus, this paper provides an experimental evaluation of the $\mathrm{n}_{2}$ and $\beta_{\mathrm{TPA}}$ coefficients on Ge-rich SiGe waveguides with different Ge concentrations (70, 80 and 90\%). The measurements have been performed at $1.58 \mu \mathrm{m}$ using a bidirectional $\mathrm{D}$-scan setup that provides a very accurate measurement of the output pulse spectral broadening induced by self-phase modulation at the waveguide output upon excitation by various incident dispersion coefficients imprinted in a picosecond pulse ${ }^{1}$. From this experimental result we have been able to model the evolution of the nonlinear Kerr index of these SiGe alloys at extended wavelengths in the mid-IR range ${ }^{2}$. To exploit the NL effects for efficient devices such as broadband supercontinuum, we then optimized the waveguide design to obtain simultaneously good mode overlap with the Ge-rich region (the one that presents the highest $\mathrm{n}_{2}$ ) and a broadband flat anomalous dispersion over a wavelength range from $\lambda=3 \mu \mathrm{m}$ to $8 \mu \mathrm{m}$. From these results it has been possible to estimate the NL parameter $\left(\gamma_{\text {eff }}\right)$ of such waveguides, suggesting a promising performance for broadband supercontinuum generation.

\section{Sample description and experimental setup}

Waveguides were defined on a $2 \mu$ m-thick Ge-rich $\mathrm{Si}_{1-\mathrm{x}} \mathrm{Ge}_{\mathrm{x}}$ guiding layer grown on a graded buffer layer $11 \mu \mathrm{m}$ thick with a linear increase of the Ge concentration to accommodate the lattice mismatch between $\mathrm{Si}$ and Ge. Three different stacks were grown, each one having a different Ge concentration on the $2 \mu \mathrm{m}$ thick Ge-rich SiGe layer: $\mathrm{x}=0.7,0.8$ and 0.9. Figure 1(a) shows a typical cross-section view of the fabricated waveguides, with an etching depth of $1 \mu \mathrm{m}$ and a waveguide width of $1.6 \mu \mathrm{m}$. Figure 1(b) displays the waveguide vertical refractive index profile employed to simulate the optical performance, providing an accurate agreement with the measured characteristics. Figure 1(c) shows the correlation between the simulated effective refractive index in the waveguide, $\mathrm{n}_{\text {eff }}$ (left $\mathrm{y}$-axis figure in black), the nonlinear effective modal area, $A_{\text {eff }}$ (right $y$-axis figure in red) and the $\mathrm{Ge}$ concentration in the $2 \mu \mathrm{m}$ thick guiding layer.

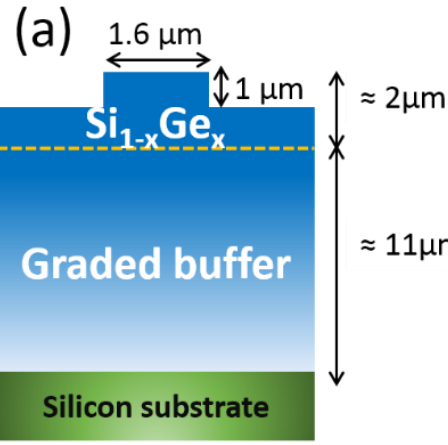

(b)



(c)



Figure 1: (a) Waveguide cross-section of the Ge-rich Si1-xGex waveguides. (b) Modeling of the vertical refractive index profile of waveguides. (c) Evolution of the simulated effective waveguide

\footnotetext{
${ }^{1}$ Samuel Serna and Nicolas Dubreuil, "Bi-directional top-hat D-Scan: single beam accurate characterization of nonlinear waveguides," Opt. Lett. 42, 3072-3075 (2017)

${ }^{2}$ S. Serna, V. Vakarin, J.-M. Ramirez, J. Frigerio, A. Ballabio, X. Le Roux, L. Vivien, G. Isella, E. Cassan, N. Dubreuil, and D. Marris-Morini, "Nonlinear Properties of Ge-rich Si1-xGex Materials with Different Ge Concentrations," Sci. Rep. 7, (2017).
} 
refractive index ( $n_{\text {eff }}$, left $y$-axis in black) and the nonlinear effective modal area $\left(A_{\text {eff }}\right.$, right $y$ axis in red) as a function of the Ge concentration ( $x$-axis).

For the nonlinear characterization, we use a mode-locked Erbium doped fiber laser that delivers $150 \mathrm{fs}$ pulses with a repetition rate of $20 \mathrm{~ns}$. A grating based pulse stretcher is used to control the spectral shape and the second order dispersion of the pulses ${ }^{3}$. The pulsed beam is injected inside the strip SiGe waveguides using a microscope objective. The injected pulse has an almost rectangular-shaped spectrum, with a linewidth of $7.3 \mathrm{~nm}$. For $\varphi^{(2)}=0 \mathrm{ps}^{2}$, the measured auto-correlation pulse duration is 2 ps, close to the Fourier-limit.

When a waveguide presents TPA, a linear relation that contains the output power $\left(P_{\text {out }}\right)$ and the input power $\left(P_{\text {in }}\right)$ can de derived as $P_{\text {in }} / P_{\text {out }}=b P_{\text {in }}+a$, with $a=1 /\left(\kappa_{\text {in }} \kappa_{\text {out }} \exp (-\alpha L), \quad b=\right.$ $2 \gamma_{T P A} \eta L_{e f f} /\left(\kappa_{\text {out }} \exp (-\alpha L) . L_{\text {eff }}\right.$ is the effective length of the waveguide and $\eta$ is a parameter that relates the average power with the peak power, being $\eta=1 /\left(F \int_{0}^{1 / F}|U(t)|^{2} d t\right)$ and $U(t)$ is the normalized temporal shape of the pulse. $\kappa_{\text {in }}$ and $\kappa_{\text {out }}$ are the coupling efficiencies, depicted in Fig.2. The spectral output measurements are done in the propagation direction $+z$ with pulses under different dispersion $\phi^{(2)}$ varying from -3.5 to $+3.5 \mathrm{ps}^{2}$. This dispersive scan changed the waveguide nonlinear response depending on the sign and magnitude of the instantaneous third order nonlinear effects. Applying four different input powers $P_{i n}$, the spectral r.m.s. width $2 \sigma_{\lambda}$ from the transmitted pulses was used to determine the sign of the Kerr coefficient and the magnitude of the figure of merit $F_{O M} M_{T P A}=$ $\frac{\gamma}{4 \pi \gamma_{T P A}}$, where $\gamma_{T P A}=\operatorname{Im}(\gamma)=b /\left(2 a \kappa_{i n} \eta L_{e f f}\right)$ and $\gamma=\operatorname{Re}(\gamma)=k_{0} n_{2} / A_{N L}$.

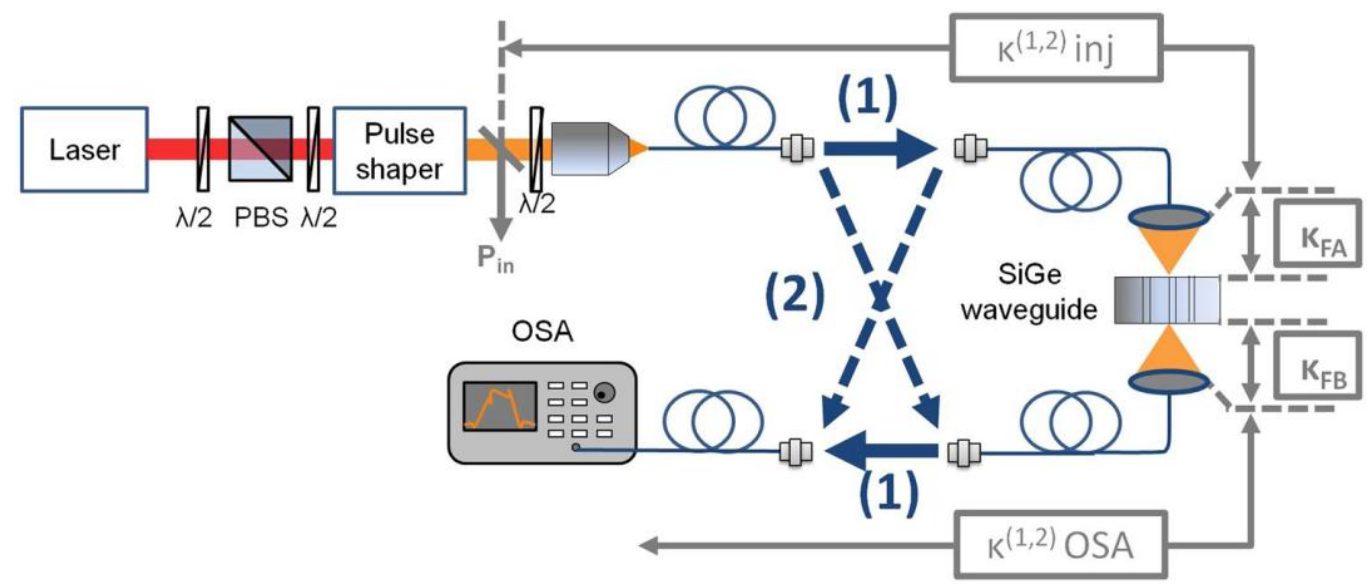

Figure 2: Experimental bi-directional setup used to measure the nonlinear parameter in Ge-rich SiGe waveguides.

\section{Experimental results and modeling of the third order nonlinear parameter}

By means of a two band model and the Kramers-Krönig (KK) relations (principle of causality), SheikBahae and co-workers ${ }^{4}$ have deduced an expression for the bound electronic nonlinear refractive index $n_{2}$ in semiconductors and wide-gap optical solids. The expression is given by:

$$
n_{2}(\omega)=\frac{40 \pi \sqrt{E_{p}}}{c n_{0}^{2} E_{g}^{4}} K^{\prime} G_{2}\left(\frac{\hbar \omega}{E_{g}}\right)
$$

\footnotetext{
${ }^{3}$ Samuel Serna, Jérèmy Oden, Marc Hanna, Charles Caer, Xavier Le Roux, Christophe Sauvan, Philippe Delaye, Eric Cassan, and Nicolas Dubreuil, "Enhanced nonlinear interaction in a microcavity under coherent excitation," Opt. Express 23, 29964-29977 (2015)

${ }^{4}$ M. Sheik-Bahae, D.C. Hutchings, D.J. Hagan, and E.W. Van Stryland, "Dispersion of bound electron nonlinear refraction in solids," Quantum Electronics, IEEE Journal of, 27(6):1296 -1309, (1991).
} 
where $E_{g}$ is the gap energy, $n_{0}$ the material index of refraction, $+E_{p}=21 \mathrm{eV}$ is the Kane energy and $K^{\prime}$ is a numerical fitting coefficient that takes values of the order of $1.5 \times 10^{-8}$ if the energies are in $\mathrm{eV}$. The function $G_{2}$ describes the contributions from different physical origins: two-photon transitions (TPA and Raman (R)), linear Stark (LS), quadratic Stark (QS) and a divergent term (DT). They are related as: $G_{2}(x)=G_{T P A}(x)+G_{R}(x)+G_{L S}(x)+G_{Q S}(x)-G_{D T}(x)$. The exact form of the equations may be found in Ref. [4]. In Figure 3, we have plotted the frequency dependent evolution of the various contributions to the nonlinear refractive index. It is interesting to notice that at the gap energies of the Ge-rich SiGe alloys, the values of $n_{2}$ are expected to strongly vary nearby $h v \approx 0.7 E_{g}$, which is the case of telecom wavelengths. So, to properly characterize and differentiate all the materials, it will be required to use a very precise method.

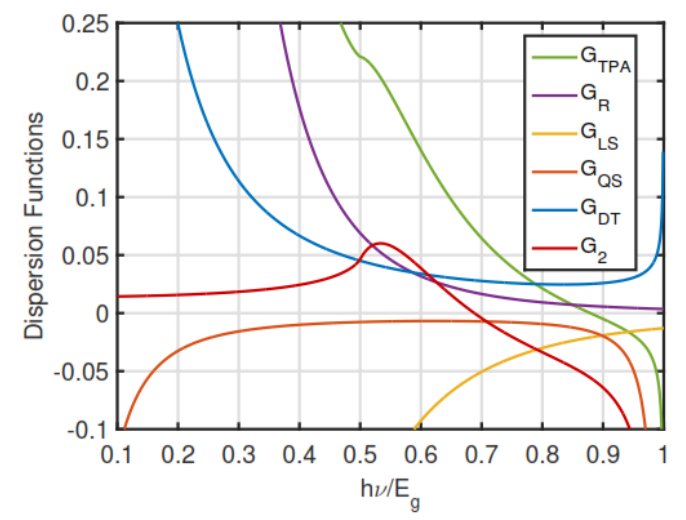

Figure 3: Frequency dependence of the various contributions to the nonlinear refractive index.

We could apply then, the bi-directional D-Scan method in order to measure the non-linear properties of the waveguides with the different Ge concentrations.. In Figure 4, we present the output spectra of the $80 \% \mathrm{Ge}$ concentration SiGe waveguide as a function of the second order dispersion parameter (DScan) for an average input power of $10 \mathrm{~mW}$. Figure 4 (a) presents the experimental results by changing the pulse shaper conditions, the symmetry in the spectral broadening is a signature of the absence of effects from free carriers generated by TPA process, so we can attribute those features to instantaneous effects. Figure 4 (b) displays the simulation results by considering the system under study as a temporal lens, i.e. the nonlinearity is considered as a phase operator that, when neglecting the dispersion introduced by the waveguide, is analogous to a thin lens system. In this particualr case, the nonlinear phase shift is equal to $0.43 \mathrm{rad}$. In Figure 4 (c) we have used the theoretical relation for the calculation of $n_{2}$ explained before.


Figure 4: (a) Experimental D-Scan obtained by changing the second order dispersion of a top-hat beam with average power of $10 \mathrm{~mW}$. (b) Simulated spectral evolution for a waveguide introducing a nonlinear phase shift of $0.43 \mathrm{rad}$. (c) Comparison of theoretically calculated $n_{2}$ dispersion curves and experimental measurements for SiGe alloys with different Ge concentrations. 


\section{Design of graded-index SiGe waveguides for mid-IR supercontinuum generation}

Figure 4 (c) shows the calculated trend for $\mathrm{n}_{2}$ for wavelengths spanning from near- to mid-IR range. As observed, despite we obtain a mild decrease of the $\mathrm{n}_{2}$ value for early mid-IR wavelengths beyond $\lambda$ $=2 \mu \mathrm{m}$, the fact that two-photon absorption vanishes for wavelengths $>3.15 \mu \mathrm{m}$ in Ge gives access to a promising 'sweet spot' to exploit the nonlinearities in the mid-IR efficiently. For that, a waveguide dispersion engineering was performed to obtain a broadband anomalous dispersion over one octave spanning, from $\lambda=3 \mu \mathrm{m}$ to $8 \mu \mathrm{m}$, while maintaining a small effective area and a good overlap with the Ge-rich area. Noticeably, the vertical graded-index refractive index profile provides an optimum mode confinement in a broadband wavelength range, re-adapting its modal size according to the propagating wavelength, as can be observed in the top panels of figure 5 for the TE mode. Due to this fact, a single waveguide geometry can provide optimum conditions to exploit the nonlinear properties over a wide wavelength range. To better depict this situation, the bottom panels of figure 5 describe the evolution of the anomalous dispersion (colored areas) as a function of the etching depth (y-axis) and the waveguide width (x-axis) for the TE mode, showing a mild displacement of the anomalous dispersion condition over one octave spanning in the mid-IR.
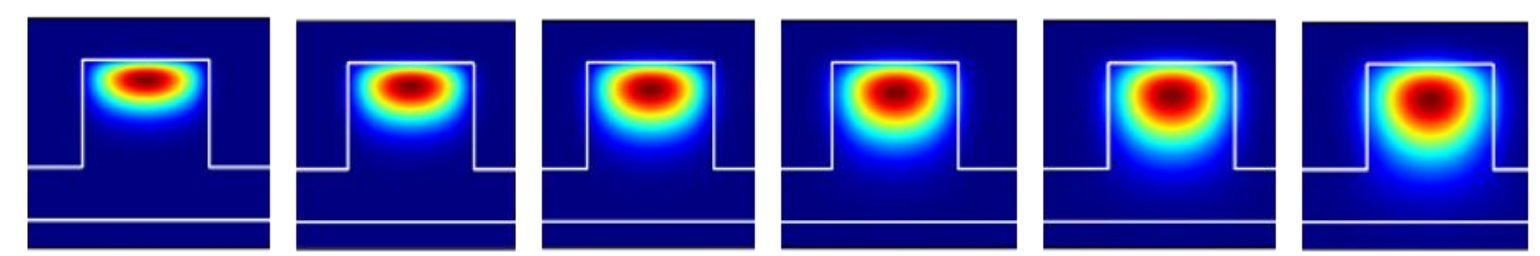

$$
\lambda=3 \mu m
$$


Figure 5: (Top panels) Modal simulations of the propagating optical mode in an optimized waveguide geometry for a broadband wavelength range from $\lambda=3 \mu \mathrm{m}$ to $\lambda=8 \mu \mathrm{m}$. (Bottom) 2D maps showing the anomalous dispersion condition (colored areas) as a function of the waveguide etching depth (yaxis) and width (x-axis).

Finally, the effective third-order nonlinear parameter $(\gamma)$ of the propagating mode has been estimated using the previously obtained $\mathrm{n}_{2}$ values and the calculated modal effective area, as shown in figure 6(a). As seen in figure 6(b), the $\gamma$ value displays a gradual decrease with longer wavelengths according to the increase of the effective area. Such values are in line with previous works that demonstrate midIR supercontinuum generation using $\mathrm{SiGe}$ waveguides with moderate Ge concentrations of $42 \%$, proving the potential of these platforms for nonlinear mid-IR photonics. 

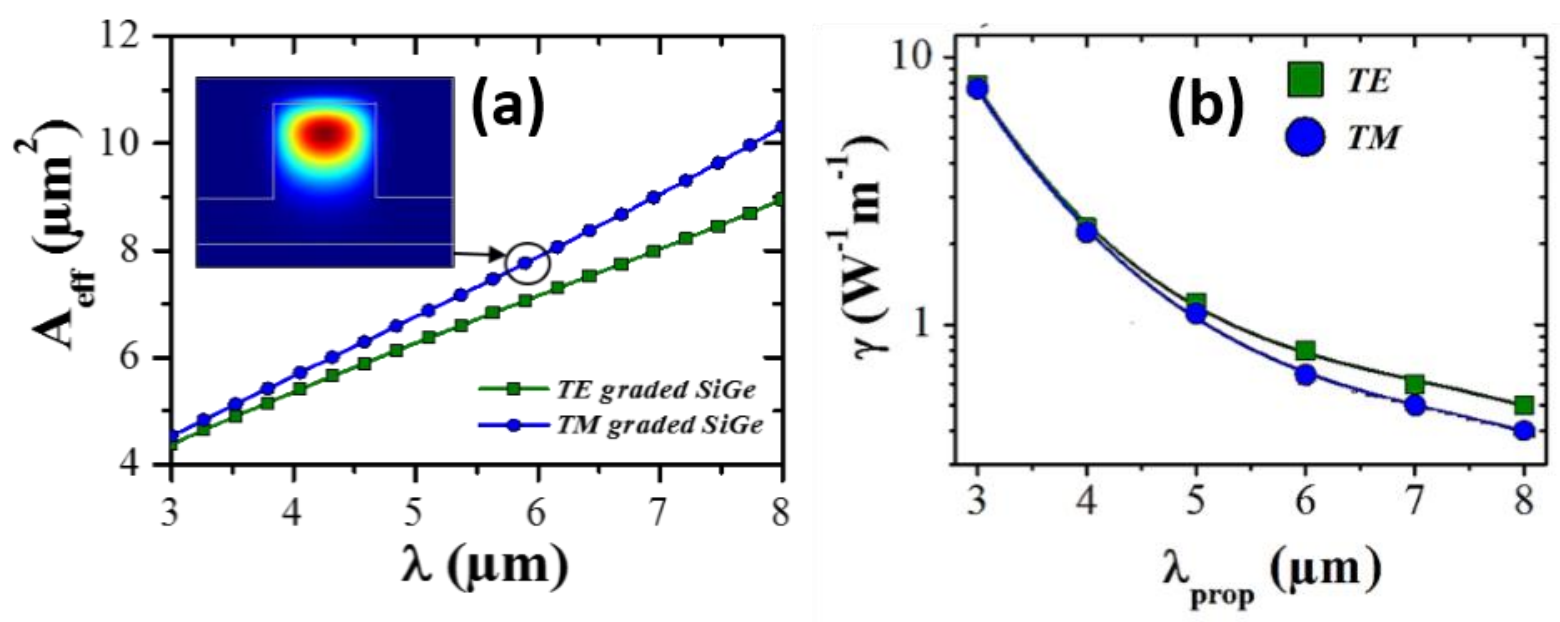

Figure 6: Evolution of the modal effective area (a) and the third-order nonlinear parameter (b) as a function of the wavelength for the TE (green squares) and TM (blue dots) polarizations.

\section{Conclusions}

To summarize, we have reported to the best of our knowledge the first experimental characterization of the instantaneous nonlinearities in Ge-rich SiGe alloy waveguides. We have presented the interest of using the SiGe platform for monolithic integration and for the exploitation of its properties in the midIR wavelength region, in particular for nonlinear integrated based applications such as supercontinuum generation, which may be a key for the development of compact and low cost midIR spectrometers.

Furthermore, we demonstrated that the nonlinear properties of SiGe waveguides could be tuned through innovative band-gap engineering, while the use of such a graded buffer grants a high quality Ge-rich SiGe layer on Si.

\section{ACKNOWLEDGMENTS}

This project has received funding from the European Research Council (ERC) under the European Union's Horizon 2020 research and innovation program (grant agreement $\mathrm{N}^{\circ} 639107-$ INsPIRE). The fabrication of the device was performed at the Plateforme de Micro-Nano-Technologie/C2N, which is partially funded by the "Conseil Général de l'Essonne". This work was partly supported by the French RENATECH network.

\section{REFERENCES}

